\title{
Combined detection of C-reactive protein and PBMC quantification from whole blood in an integrated lab-on-a-disc microfluidic platform
}

\author{
Uddin, Rokon; Donolato, Marco; Hwu, En Te; Hansen, Mikkel Fougt; Boisen, Anja
}

Published in:

Sensors and Actuators B: Chemical

Link to article, DOI:

10.1016/j.snb.2018.07.015

Publication date:

2018

Document Version

Peer reviewed version

Link back to DTU Orbit

Citation (APA):

Uddin, R., Donolato, M., Hwu, E. T., Hansen, M. F., \& Boisen, A. (2018). Combined detection of C-reactive protein and PBMC quantification from whole blood in an integrated lab-on-a-disc microfluidic platform. Sensors and Actuators B: Chemical, 272, 634-642. https://doi.org/10.1016/..snb.2018.07.015

\section{General rights}

Copyright and moral rights for the publications made accessible in the public portal are retained by the authors and/or other copyright owners and it is a condition of accessing publications that users recognise and abide by the legal requirements associated with these rights.

- Users may download and print one copy of any publication from the public portal for the purpose of private study or research.

- You may not further distribute the material or use it for any profit-making activity or commercial gain

- You may freely distribute the URL identifying the publication in the public portal 


\section{Accepted Manuscript}

Title: Combined detection of C-reactive protein and PBMC quantification from whole blood in an integrated lab-on-a-disc microfluidic platform

Authors: Rokon Uddin, Marco Donolato, En-Te Hwu, Mikkel Fougt Hansen, Anja Boisen

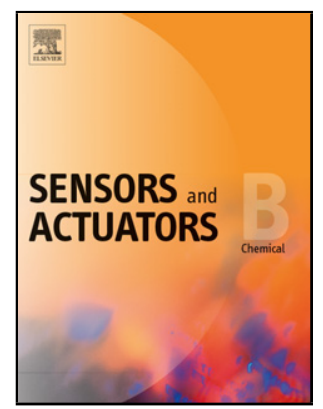

PII: S0925-4005(18)31248-6

DOI: https://doi.org/10.1016/j.snb.2018.07.015

Reference: SNB 24984

To appear in: Sensors and Actuators B

Received date: 2-2-2018

Revised date: 3-6-2018

Accepted date: 2-7-2018

Please cite this article as: Uddin R, Donolato M, Hwu E-Te, Hansen MF, Boisen A, Combined detection of C-reactive protein and PBMC quantification from whole blood in an integrated lab-on-a-disc microfluidic platform, Sensors and Actuators: B. Chemical (2018), https://doi.org/10.1016/j.snb.2018.07.015

This is a PDF file of an unedited manuscript that has been accepted for publication. As a service to our customers we are providing this early version of the manuscript. The manuscript will undergo copyediting, typesetting, and review of the resulting proof before it is published in its final form. Please note that during the production process errors may be discovered which could affect the content, and all legal disclaimers that apply to the journal pertain. 


\title{
Combined detection of $\mathrm{C}$-reactive protein and PBMC quantification from whole blood in an integrated lab-on-a-disc microfluidic platform
}

\author{
Rokon Uddin, ${ }^{\mathrm{a}}$ Marco Donolato, ${ }^{\mathrm{b}}$ En-Te Hwu, ${ }^{\mathrm{a}}$ Mikkel Fougt Hansen, ${ }^{\mathrm{a}}$ and Anja Boisen ${ }^{\mathrm{a}}$
}

a Department of Micro- and Nanotechnology, Technical University of Denmark, DTU Nanotech, Building 345B, DK-2800 Kongens Lyngby, Denmark

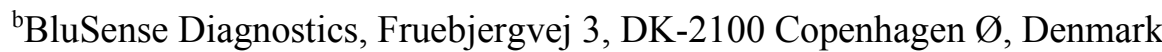

*Corresponding author: Rokon Uddin

Contact: Ørsteds Plads, Building 345C, 110

2800 Kgs. Lyngby, Denmark

Tel: +4545256343

Email: rokud@nanotech.dtu.dk

Highlights

- A biosensing platform to concurrently detect CRP and PBMC from single blood sample

- The microfluidic chip/disc and the readout units are integrated in a single platform

- $\quad$ The entire assay procedure is automated from sample to answer

- The biosensor needs low sample volume and low sample-to-answer time

- It has potential characteristics to be implemented as an out-of-lab setting

\begin{abstract}
There is an increasing need for portable and low-cost diagnostic devices for detecting inflammatory/infectious diseases in a rapid and user-friendly fashion. Here, we present a lab-on-a-disc solution, which performs automated sample pretreatment and combinedly detects small molecules and counts cells in a whole blood sample with a volume of $8.75 \mu \mathrm{L}$ with a sample to answer time of $14 \mathrm{~min}$. It is used to detect two common inflammation/infection biomarkers, C-reactive protein (CRP) and peripheral blood mononuclear cell (PBMC) count. The whole blood sample was separated into plasma and PBMC fractions using density gradient centrifugation and centrifugo-pneumatic valving. On-disc CRP detection was performed in the extracted plasma using a CRP-antibody-functionalized magnetic nanobead (MNB)based agglutination assay and a Blu-ray-based optomagnetic detection unit. On-disc PBMC scanning and quantification was performed using an optical imaging unit. Both detection units were integrated on the centrifugal platform and the entire study was automated in order to ensure reliability of the assay and user-friendliness of the method. We measured the CRP level of subjects with different CRP levels and obtained approximately 73\% PBMC extraction efficiency compared to hospital results. The concurrent/combined detection of these two common biomarkers in an automated
\end{abstract}


microfluidic platform with integrated detection units and with a low sample-to-answer time is a significant step forward towards a low-cost, out-of-lab, and portable tool to detect multiple biomarkers of significantly different nature (molecules and cells).

Keywords: CRP, PBMC, centrifugal microfluidics, magnetic beads, optomagnetic readout, optical imaging

\section{Introduction}

CRP is a protein found in blood plasma and its level increases significantly in response to most inflammations. It is a widely used biomarker for detecting acute-phase inflammation, infection and tissue damage [1]-[3]. Additionally, an increase in plasma CRP level indicates a higher risk of myocardial infarction, atherosclerosis or other coronary diseases [4]. The median value of CRP in plasma from healthy adults is $0.8 \mathrm{mg} / \mathrm{L}$ [1], concentrations above $3 \mathrm{mg} / \mathrm{L}$ indicate a high risk of cardiovascular diseases [4] and in the case of infections, the level can rise to more than 500 mg/L [1]. However, a CRP test is non-specific in nature [5]. Therefore, it is necessary to measure other relevant biomarkers along with CRP to perform a specific diagnosis or an advanced prognosis. One of such biomarkers is the white blood cell (WBC) count or peripheral blood mononuclear cell (PBMC) count, which are also known to increase in response to most inflammations or infections [6]-[7]. WBCs include five different cell types: lymphocytes, monocytes and granulocytes (eosinophil, basophil and neutrophil), where the former two mononuclear cells (lymphocytes and monocytes) are the PBMCs. The total WBC count, the PBMC count, and the count of each of the individual type of WBCs are markers for different diseases and medical conditions. In addition to inflammation/infections, elevated levels of WBCs have been associated with increased risk of mortality among patients with coronary heart disease or patients undergoing haemodialysis. Furthermore, type-2 diabetes, HIV, different types of cancer, immune system disorder and even hypertension have been reported to increase the WBC count [8]-[14].

Studies have found positive correlations between the WBC or PBMC counts and different inflammatory biomarkers including CRP [10], [13] for most inflammations and certain bacterial infections such as pneumococcal pneumonia [15]. It has also been suggested that PBMCs themselves may participate in the production of CRP in blood [16], hence indicating a strong correlation between the PBMC count and CRP concentration for the prognosis of certain diseases. Furthermore, epidemiologic studies have shown that an elevated CRP level in apparently healthy individuals can be a marker of high potential risk of certain types of cancer and that a high CRP level in cancer patients indicates a risk of earlier death by cancer [17]. PBMCs have also been linked to cancer prognosis [18]. Thus, while CRP tests act as the first-line screening for various infectious and inflammatory diseases as well as cancer and cardiovascular conditions, combining CRP detection with WBC or PBMC count and further cell analysis may lead to an improved diagnosis and prognosis.

In clinical laboratories, the CRP concentration is determined using a latex-enhanced immunoturbidimetric assay [19], [20] on a human serum sample exposed to a number of sample pre-treatment steps. In the literature, different approaches to determine the CRP concentration in human serum have been presented including a magnetic bead-based agglutination assay [21]-[23], quantum dots and immunofiltration [24], quantum dots and immunochromatography [25], 
electrochemical aptamer-based sandwich assay [26], SPR-based immunosensing using CRP antibodies

[27], nanoparticle-enhanced SPR using aptamer-antibody sandwich assay [28] as well as fluorescence-based biosensing [29]. Furthermore, Gyros Protein Technologies (Sweden) uses microfluidic discs (Gyrolab CD) to integrate sample pretreatments minimizing manual interventions to quantify CRP using a nanoliter-scale sample volume. However, separate microfluidic discs are needed to detect different biomarkers and it does not combine an immunoassay with WBC count.

WBC or PBMC counts are determined in standard clinical laboratories by manual or automatic counting. The manual counting is performed in a haemocytometer using standard microscopy. The automatic counting is commonly done by flow cytometry or automated image cytometry [30]. All these methods all require a number of complex and timeconsuming sample pre-treatment steps, including fluorescent labelling, staining and red blood cell (RBC) lysis prior to the actual counting. The widely available point-of-care device HemoCue-WBC (HemoCue AB) can perform WBC counting with high accuracy with no need for sample pre-treatment. However, as the device has no integrated optical imaging unit, it is not possible to investigate physical characteristics of the cells, study cell agglutination or to analyse their morphologic abnormalities. Furthermore, WBC count alone is insufficient for the diagnosis of a disease with full certainty. There are reports on cell analysis and counting in centrifugal microfluidics platform [31]-[33], but these studies lacked an integrated sample-to-answer platform for cell counting as well as fell short of demonstrating concurrent detection of other relevant biomarker(s) for prognosis of a disease. Similarly, Biosurfit's (Portugal) Spinit ${ }^{\circledR}$ instrument uses surface plasmon resonance to perform an immunoassay and its integrated microscopy module counts WBCs on a microfluidic disc. However, separate microfluidic platforms/discs are needed to perform these two operations and thus it does not perform a combined detection/quantification of the two biomarkers from a single blood sample. Likewise Hemocue-WBC, the Spinit ${ }^{\circledR}$ instrument lacks the imaging display to investigate physical characteristics of cells. Thus, in spite of biosensors having achieved significant success in diagnostics [34], there is an unmet need for a portable and automated diagnostic tool that in a single microfluidic platform combines detection of relevant biomarkers with the WBC/PBMC count for advanced prognosis of inflammatory/infectious diseases.

Here, as a first but significant step forward, we present an integrated platform to combinedly detect the concentration of CRP and quantify PBMCs directly from few microliters of human whole blood, where a single low-cost microfluidic disc is used as the experimental platform onto which all assay steps are integrated and automated. First, the density gradient centrifugation method [35] is used for on-disc plasma and PBMC separation. Then, the separates are transferred to distinct microfluidic chambers for further on-disc processing.

The CRP in the plasma is detected using a sandwich agglutination assay employing magnetic nanobeads (MNBs) [23], [36]-[43]. To promote and accelerate agglutination of MNBs in the presence of CRP, we use a magnetic field incubation protocol [41], [44]. Agglomerates are detected using a Blu-ray based optomagnetic readout technique [39][41], which measures the modulation of light transmitted through the sample in response to an applied oscillating magnetic field as function of the frequency of the magnetic field. The individual MNBs are superparamagnetic and substantially spherical and therefore show nominally no signal. Agglomerated MNBs have coupled magnetic and optical anisotropies and therefore show a signal at low frequencies where they can rotate and thereby modulate the intensity of transmitted light in response to the applied oscillating magnetic field. The on-disc PBMC counting is performed on the PBMC separate using an integrated optical imaging unit (oCelloScope, BioSense Aps) [40], [45], 
which is an automated bright-field microscope that uses optical sectioning from confocal microscopy to create a volume image. This makes it possible to capture images of all cells in the sample. The PBMCs are identified and quantified using the instrumental software Uniexplorer 8.0, that employs an object-based image analysis algorithm to segment the cells based on their surface area.

Both detection units (optomagnetic reader and optical volume imaging) are integrated with the automated centrifugal platform facilitating a compact sample-to-answer device for detection of CRP and PBMC quantification directly on a single whole blood sample in a fast, reliable and user-friendly fashion while using a low-cost polymer microfluidic disc consumable. The design and the automated operation of the microfluidic disc to extract PBMCs and a certain amount of plasma to separate microfluidic chambers, the integration of the hardware of the two detection units along with the automated centrifugal microfluidic platform, the on-disc detection of CRP on the extracted plasma sample and on-disc PBMC counting from a single blood sample within a single experiment demonstrate the inherent advantages of the integrated biosensing platform to combinedly detect biomarkers of different sizes or nature.

\section{Experimental}

\section{1 Materials and chemicals}

The experimental platform of this study was a Poly(methylmethacrylate) (PMMA)-based microfluidic disc fabricated in-house. The blood samples (EDTA-treated), collected from healthy donors, were purchased from the Copenhagen University Hospital (Rigshospitalet). For efficient separation between the RBCs and the PBMC layer, a density gradient medium (Histopaque-1077, Sigma-Aldrich) was used during blood processing. The MNBs used in this study were carboxy-labelled particles (Millipore Estapor-Merck, Product number: M1-020/50) with an average diameter of $176 \mathrm{~nm}$. These MNBs are nominally spherical and superparamagnetic and thus show no optomagnetic response when they are not agglomerated. The MNBs were functionalized with goat polyclonal CRP antibodies (Midland Bioproducts, USA, Product number: 73307) to enable the agglutination of the MNBs in presence of the CRP antigen in the blood plasma. We have used this affinity-purified CRP antibody because of its well-proven specificity to bind with CRP molecule [21]. CRP-free serum (Hytest Ltd., Turku, Finland) was used for sample dilution and negative control experiments. 10 mM PBS (pH 7.4) buffer was prepared using MilliQ water for MNB suspension and blood dilution.

\subsection{Functionalization of magnetic nanobeads with CRP antibodies}

The magnetic nanobeads (MNBs) were functionalized with CRP antibodies following a previously described protocol [41]. This protocol defines an anti-fouling surface architecture over the MNBs utilizing bio-orthogonal click chemistry to minimize the formation of non-specific aggregates under the influence of complex biological fluids. Thus, a monolayer of blocking proteins - human serum albumin HSA (Sigma Aldrich, A9511) was firstly immobilized on the MNBs by means of carbodiimide chemistry. $50 \mathrm{mg}$ of HSA was added to $500 \mu \mathrm{L}$ of MNB sample at a bead concentration of $5 \%(\mathrm{w} / \mathrm{v})$ followed by overnight incubation. The MNBs were then washed three times and resuspended in $500 \mu \mathrm{L}$ of PBS buffer with $0.1 \%$ Pluronic F-127 (PBSP). Next, 1.25 mg of NHS-PEG4-N3 (ClickChemistryTools, 
AZ103-25) linker was added to a sample of $500 \mu \mathrm{L}$ HSA-coated MNBs and incubated at room temperature for 1 hour. The MNB solution was then washed three times with PBSP and resuspended at a final volume of $500 \mu \mathrm{L}$. This ensured the amine-reactive linker to covalently bind to HSA which facilitated the introduction of azide $\left(\mathrm{N}_{3}{ }^{-}\right)$moieties on the MNB surface.

To functionalize the azide-linked MNBs with the antibodies, azide-complementary moieties were needed to be added to the antibodies. Hence, the goat polyclonal CRP antibodies were buffer exchanged to PBS followed by adding a 20 -fold molar excess of azide-complementary NHS-PEG4-DBCO (ClickChemistryTools, A134-10) linker to the antibodies and was incubated for 1 hour at room temperature. Click reaction was performed between the coated MNBs and the antibodies at $37^{\circ} \mathrm{C}$ for 3 hours to ensure the functionalization of MNBs with the antibodies through the bonding between azide moiety and azide-complementary DBCO. After the reaction, the MNB solution was washed three times with PBSP and finally resuspended at a volume of $500 \mu \mathrm{L}$ for performing the experiments.

\subsection{Microfluidic disc fabrication}

All experiments were performed using a PMMA-based microfluidic disc onto which the entire assay from sample preparation to CRP detection and PBMC quantification was integrated. Each disc consisted of six repeating operational units enabling six simultaneous experiments (Fig. 1a). The disc was fabricated through bonding of three different PMMA layers and one PSA (pressure sensitive adhesive) layer (Fig. 1b). The top PMMA layer (thickness $0.6 \mathrm{~mm}$ ) consisting of vent and load holes was thermally bonded to the middle PMMA layer (thickness $1.2 \mathrm{~mm}$ ) housing microfluidic channels and chambers using a thermal bonding press (PW 10 H, P/O/Weber GmbH, Germany). The thermal bonding of the PMMA layers housing microchannels eliminated the need for using PSA for bonding and ensured fabrication reproducibility; because patterning narrow microchannels in PSA layer followed by peeling them (sticky microchannels) off from the layer is challenging and time-consuming as well as it introduces fabrication tolerances. For thermal bonding, the two layers were aligned together on an aluminium plate (thickness: $1 \mathrm{~mm}$ ) and bonded with $14 \mathrm{kN}$ force at $85^{\circ} \mathrm{C}$ temperature for $10 \mathrm{~min}$. The holes and the chambers were laser-cut and the channels were laser-ablated using a $\mathrm{CO}_{2}$ laser (Epilog Mini 18, 30W, Epilog, USA). Next, a PSA layer (AR7840, Adhesives Research Ltd, Ireland; thickness: $86 \mu \mathrm{m}$ ) accommodating the microfluidic chambers with an additional chamber for accommodating the PBMCs was used to bond the top and middle layers to the base PMMA layer. This cell counting (CC) chamber was defined in the thin PSA layer to ensure a monolayer distribution of the PBMCs (resembling the CC chamber of a standard haemocytometer) for the optical scanning. The total fabrication time for a single disc was approximately $35 \mathrm{~min}$.

\subsection{Experimental procedure}

A custom-built integrated experimental platform was used for the study. The setup consisted of a microfluidic disc attached to a closed-loop motor (No. 273756, Maxxon Motor, Switzerland) for centrifugation, blood-processing and disc-positioning, a pair of cylindrical $(\varnothing 7 \mathrm{~mm}$, thickness: $2 \mathrm{~mm})$ permanent magnets $(B=60 \mathrm{mT}$ at the centre) to perform on-disc magnetic incubation [40], [41], a Blu-ray based optomagnetic unit to detect CRP concentration using 
MNB-based agglutination assay and an optical imaging unit for automated scanning and quantification of the PBMCs (Fig. 2a). The entire study was automated in order to facilitate reproducibility and user-friendliness of the assay. The complete experimental procedure is shown in the Supplementary Movie.

After mounting the disc, $35 \mu \mathrm{L}$ of density gradient media (DGM) (Histopaque-1077, Sigma-Aldrich) was loaded into the loading chamber of an operational unit (Fig. 2b) of the disc followed by centrifugation at $25 \mathrm{~Hz}$ to guide the DGM to the blood processing chamber (Figs. 2c-i). Next, $8.75 \mu \mathrm{L}$ of EDTA-treated human whole blood was diluted in PBS buffer with a volume ratio 1:4 for effective isolation of PBMCs. The diluted blood sample (35 $\mu \mathrm{L})$ was loaded using high-speed centrifugation $(45 \mathrm{~Hz})$ with a low acceleration $(2.5 \mathrm{~Hz} / \mathrm{s})$ rate causing the blood to first overlay on the DGM (Fig. 2c-ii) followed by stratification into plasma, a PBMC layer and RBCs within 4 minutes (Fig. 2c-iii). The mononuclear cells (PBMCs) consisting of $40 \%$ of the total WBCs were less dense than the DGM and therefore formed a layer at the plasma-DGM interface. The RBCs being aggregated by the polysucrose (present in the DGM) sedimented at the bottom of the blood-processing chamber along with the other parts of WBCs (i.e. granulocytes).

The air inside the blood-processing chamber was compressed when the DGM and the blood entered the processing chamber followed by the high-speed centrifugation. Thus, after the blood-component stratification, decreasing the centrifugation speed to $10 \mathrm{~Hz}$ with a low de-acceleration rate $(2.5 \mathrm{~Hz} / \mathrm{s})$ caused the compressed air to expand gradually pushing the PBMC layer, plasma and some DGM into the cell counting (CC) and plasma-holding (PH) chambers via the upper siphon (Figs. 2c-iv). The basic mechanism of this centrifugo-pneumatic valving can be found in [46]. The inlet of the upper siphon was positioned so that it extracted approximately $34 \mu \mathrm{L}$ from the blood processing chamber. The de-acceleration was paused at $10 \mathrm{~Hz}$ for $30 \mathrm{~s}$ to enable PBMCs to flow down from the PH chamber to the CC chamber. Thus, the CC chamber was filled with $26 \mu \mathrm{L}$ of liquid with PBMCs and the PH chamber held approximately 8 $\mu \mathrm{L}$ of plasma. Due to the large difference between the heights of the PH chamber (height: $1.28 \mathrm{~mm}$ ) and the CC chamber (height: $86 \mu \mathrm{m}$ ), the plasma in the $\mathrm{PH}$ chamber experienced an increased pressure at the junction between the two chambers [47]. In addition, when the liquid entered the CC chamber it entrapped and compressed the air inside the chamber. Thus, when the centrifugation speed was reduced from $10 \mathrm{~Hz}$ to $2.5 \mathrm{~Hz}$, the pressure on the plasma at the junction was reduced and the compressed air expanded, which subsequently enabled the plasma in the PH chamber to prime the lower siphon and enter the plasma chamber (Fig. 2c-v). The CC chamber thus provides double-advantage to the assay i.e. assists in centrifugo-pneumatic pumping and allows monolayer distribution of the cells. Finally, the PBMCs with some amount of plasma and DGM stayed in the CC chamber and approximately $8 \mu \mathrm{L}$ of plasma remained in the plasma chamber.

After that, $22 \mu \mathrm{L}$ of CRP antibody-functionalized MNBs (suspended in PBS buffer) was added to the plasma chamber followed by 3 min of magnetic incubation using the permanent magnets to enhance agglutination between antibodyfunctionalized MNBs and CRP as well as homogenous mixing of the plasma and MNBs. The magnetic incubation protocol consisted of repeated cycles of two steps: stationary incubation between the permanent magnets for $5 \mathrm{~s}$ to enhance cluster formation, followed by $3 \mathrm{~s}$ of high-speed $(20 \mathrm{~Hz})$ shaking (half-circle rotation in clockwise and anticlockwise direction) to break the unspecific MNB clusters and facilitate mixing. Further details of the magnetic incubation protocol can be found in [41]. Following the magnetic incubation, the plasma chamber was positioned under the optomagnetic unit by the motor-control software to detect the concentration of CRP in the sample by the optomagnetic readout method. Then, the CC chamber was positioned between the light source and camera of the optical 
imaging unit to image all cells present in the chamber. The software associated with the optical imaging unit controlled the stage movement of the imaging unit as well as the obtained images were segmented and the PBMCs were quantified using the instrument software. The entire experiment from sample loading to detection took approximately 14 min.

\section{Results and Discussion}

\subsection{CRP detection using optomagnetic readout}

We used the optomagnetic reader unit to detect the concentrations of CRP in blood samples from three individual subjects. The CRP levels in the blood samples were initially analysed at Hvidovre hospital (Copenhagen, Denmark) by a standard method using latex particle-based agglutination assay [48]. From the standard clinical measurement, the CRP levels of Subject 1, Subject 2 and Subject 3 were found as $1.0 \mathrm{mg} / \mathrm{L}, 2.7 \mathrm{mg} / \mathrm{L}$ and $1.6 \mathrm{mg} / \mathrm{L}$, respectively. To verify the specificity of the MNB-based assay, three sub-samples corresponding to different CRP concentrations of each individual sample were prepared on-disc. The sub-samples were made by extracting three different volumes $(8 \mu \mathrm{L}, 6 \mu \mathrm{L}$ and $4 \mu \mathrm{L}$ ) of plasma to the plasma chamber followed by addition of CRP-free serum (except $8 \mu \mathrm{L}$ sample) to obtain a constant total volume of $8 \mu \mathrm{L}$ of each sub-samples while varying only the sample concentration. The extraction of different amounts of plasma samples to the plasma chamber was ensured by varying the initial loading volume $(31 \mu \mathrm{L}-$ $35 \mu \mathrm{L}$ ) of blood sample. This approach facilitated the measurement of CRP antigen on the same sample at different plasma concentrations. Control experiments were carried out on CRP-free serum.

Mixing of CRP antibody-functionalized MNBs with the extracted plasma sample followed by magnetic incubation led to the formation of CRP-bridged MNB clusters (Fig. 3). The signal obtained by the optomagnetic unit vs. frequency of the applied magnetic field reflects the hydrodynamic size of the MNB clusters. Details of the optomagnetic readout method can be found in [39]-[41]. In brief, the optomagnetic unit consists of three basic functional sub-units: a Blu-raybased optical pickup unit emitting light at $405 \mathrm{~nm}$ wavelength, a photodetector, and a pair of electromagnets to generate AC magnetic field which induces rotation of the MNB clusters as they are magnetically anisotropic. The intensity of transmitted light is modulated by the rotation of the MNB clusters as the clusters also have an optical anisotropy, which is linked to the magnetic anisotropy. As this signal modulation depends only on the magnitude but not the sign of the applied magnetic field, the signal due to MNB clusters is detected in the second harmonic of the photodetector signal with respect to the field excitation. For a sinusoidal applied magnetic field, it can be shown that the relevant component is the in-phase second harmonic signal, $V_{2}^{\prime}$ that shows a peak at a frequency related to the Brownian relaxation frequency of the MNB cluster, which is inversely proportional to the hydrodynamic volume of the cluster [39]-[41]. Moreover, to compensate for possible variation of the intensity of the light emitted by the laser, all measurements were normalized by the zero'th harmonic signal, $V_{0}$. Thus, when no MNB clusters are formed, nominally no optomagnetic signal is observed and upon formation of MNB clusters a peak in the $V_{2}^{\prime} / V_{0}$ signal is observed at a position which is related to the inverse hydrodynamic size of the clusters and with a magnitude proportional to the number of MNB clusters.

We performed triplicate measurements for each sub-sample where the $8 \mu \mathrm{L}, 6 \mu \mathrm{L}$ and $4 \mu \mathrm{L}$ of extracted plasma correspond to respectively $100 \%, 75 \%$ and $50 \%$ of the known CRP values. Fig. $4 \mathrm{a}$, b and c show the $V_{2}^{\prime} / V_{0}$ spectra for 
the three subjects. These results indicate that a higher CRP concentration caused an increase of the peak value of the signal. A dose-response curve (Fig. $4 \mathrm{~d}$ ) was obtained by measuring the average $V_{2}^{\prime} / V_{0}$ value at $f=2.6$ to $7.5 \mathrm{~Hz}$, which corresponds to the spectral data points sensitive to different sizes of MNB-clusters.

The dose-response curves (Fig 4d) corresponding to Subject 1 and Subject 3 showed a linear dependence on the volume of extracted plasma from the subjects and for the investigated samples, the slope of the optomagnetic signal vs. volume of extracted plasma was higher for the samples with higher CRP concentration. Fig. 4e shows the average optomagnetic signal vs. the independently measured CRP concentration for the undiluted $8 \mu \mathrm{L}$ samples as well as for CRP free serum. A close to linear dependence is observed, although with a significant offset due to unspecific particle clustering. The latter makes it difficult to realize the true potential of the method and its optimization is a topic for future work. The dashed line shows the signal from the CRP-free serum plus three times its standard deviation, which corresponds to an estimated limit of detection (or concentration resolution) of about $30 \mu \mathrm{g} / \mathrm{L}$.

In future work, the assay needs to be optimized to reduce the unspecific particle clustering and to explore the doseresponse curve at a larger range of CRP concentrations. Potentially, the assay sensitivity may be increased by use of small recombinant antibodies as these possess more capture sites resulting in detection of the presence of biomolecules in minute concentrations [49].

\subsection{PBMC segmentation and quantification}

The segmentation and quantification of the mononuclear cells (PBMCs) were performed using the optical imaging unit together with the instrument software Uniexplorer 8.0. Due to centrifugation, the cells were randomly distributed at the bottom-half of the CC chamber, which was verified by scanning the entire CC chamber. Thus, the bottom-half of the $\mathrm{CC}$ chamber was the designated scanning window (surface area: $164 \mathrm{~mm}^{2}$ ) for cell imaging, which was scanned and defined by Uniexplorer for all experiments to ensure reproducibility of the scanning positions. As the CC chamber was about the thickness of $86 \mu \mathrm{m}$, the PBMCs were distributed in a monolayer, which facilitated the software to capture all the PBMCs within a single focal plane.

Due to the presence of some amount of plasma in the CC chamber and due to RBC contamination during PBMC extraction, many platelets and some RBCs were visible in the raw images (Fig. 5a). However, due to much smaller size $(2 \mu \mathrm{m})$ of the platelets compared to the PBMCs $(7-30 \mu \mathrm{m})$, the platelets could be eliminated based on the 2D surface area of the cells. However, due to size-overlapping of RBCs $(6-8 \mu \mathrm{m})$ and small lymphocytes $(7-8 \mu \mathrm{m})$, some RBCs were still visible in the segmented images (Fig. 5b). Based on the bioconcave shapes of the RBCs, they were identified and eliminated from the segmented images resulting in only PBMCs to remain for counting. Thus, from the number of segmented images provided by software, we obtained a total PBMC count (Fig. 5c).

Fig. 5d demonstrates the PBMC count/ $\mu \mathrm{L}$ of samples from three different healthy subjects. The PBMCs of the three subjects were measured with SYSMEX using flow cytometry at Rigshospitalet. From the standard clinical measurement, the PBMC counts of Subject 1, Subject 2 and Subject 3 were found as 1640, 2200 and 2240 cells/ $\mu 1$ of blood, respectively. Comparing to the hospital data, it shows that our method can extract $73 \pm 2 \%$ of the total PBMCs. 
All experiments were performed in triplicate. The small error bars from the triplicate measurements demonstrate the reproducibility of the PBMC layer extraction and quantification approach. Although the extraction efficiency falls $27 \%$ below the hospital data, the high reproducibility of the method for all the three subjects demonstrates its potential to be a reference PBMC counter.

A possible reason for the lower extraction efficiency compared to the hospital data could be that during the PBMC extraction from the blood processing chamber, a small amount of PBMCs tend to stay back in the chamber. This phenomenon has also been experienced by Kinahan et al. [50] where an on-disc DGM-based centrifugation method was used for plasma and PBMC separation followed by out-of-disc extraction for further processing and quantification of the PBMCs. However, in that study, it was demonstrated that a significant amount of PBMCs remained in the blood processing chamber resulting in an extraction efficiency of $34 \%$ compared to benchmark hospital data. Our method showed much higher efficiency, probably because of pre-dilution of the blood at a higher ratio (1:4) than the ratio (1:1) used in [50] causing better cell isolation as well as preventing cell aggregation. However, in future work different dilution factors could be investigated in order to obtain detailed understanding on the relationship between dilution factor and PBMC extraction efficiency. In addition, the manual extraction of WBCs from the disc used in [50] might have caused loss of cells whereas on-disc quantification in our approach demonstrates lower cell loss due to the integration and automation of the process. Another potential cause of the $27 \%$ lower efficiency of our approach compared to hospital data might be the limitation of the DGM method for PBMC extraction as reported in [51]. However, Moen et al. [32] demonstrated a DGM-based centrifugation approach where the WBCs, after centrifugation, were trapped inside a trough-like section in the blood processing chamber. The on-disc imaging of the trapped WBCs inside the blood processing chamber using an external microscope resulted in $95 \%$ total WBC count. But, as their study lacked detection of CRP or other relevant biomarkers, they did not need to utilize centrifugo-pneumatic pumping to extract plasma and PBMC to separate chambers through siphons, which in our case the pumping caused some PBMCs to lag behind in the blood processing chamber causing less PBMC count. Thus, we were faced with this particular limitation while combining the detection or quantification of these two biomarkers. Furthermore, in some cases, few PBMCs were observed to be at a close proximity or aggregating with each other which caused, e.g., two aggregated PBMCs to be counted as one due to the limitation of the segmentation algorithm and thus, reducing the number of PBMCs segmented. We are currently investigating different microfluidic approaches, while integrating necessary purification steps, as well as further developing the segmentation algorithm to address this issue and thereby increase the efficiency of total PBMC extraction and quantification.

\section{Conclusion}

We have presented a biosensing platform with integrated optical and optomagnetic sensors to detect the CRP concentration and to quantify PBMC from a single human whole blood sample in a quick, automated and user-friendly fashion while using a single microfluidic disc. The integration of the sensors as well as the fully-integrated lab-on-adisc assay shows potential to be a compact instrument for use in out-of-lab setting for medical diagnostics. This biosensing platform can be further applied to detect various biomarkers using MNB-based agglutination assay in combination with WBC/PBMC quantification, for example, diagnosing infectious diseases such as tuberculosis or 
medical conditions like myocardial infarctions. A significant addition to future studies could be detection of Troponin I along with CRP by splitting the extracted plasma into two different subsamples on the disc. Apart from medical diagnostics, it can also be used in food safety and environmental monitoring applications by utilizing the microfluidics for sample processing, the optomagnetic unit for detection of small molecules and the optical imaging unit for detecting presence and/or growth of microbes.

In this study, the major factors that led to the combined detection or quantification of CRP and PBMC are: (i) the microfluidic design involving siphon-based two-step centrifugo-pneumatic valving process to extract PBMCs and certain amount of plasma from few microliters of blood into separate microfluidic chambers, (ii) the hardware integration of the two detection units along with the automated centrifugal microfluidic platform and (iii) the development of MNB-based assay to detect CRP in biological fluid using the integrated optomagnetic reader unit as well as the fabrication of the microfluidic disc involving a thin CC chamber facilitating on-disc capturing of all PBMCs distributed in a monolayer using the optical imaging unit. Furthermore, the magnetic incubation process enhanced the agglutination process, decreasing the assay time for CRP detection. Using the blood samples from three individual subjects with different CRP levels, the study demonstrated increment of optomagnetic signal peaks with increasing CRP concentration. However, for the diluted sub-samples, subject-to-subject variation was experienced for the corresponding values of the optomagnetic signal peaks. Our future work aims to optimize the MNB-based assay to minimize the subject-to-subject variation to enable a quantitative analysis with a well-established limit of detection and dynamic range of the agglutination assay while using a higher number of clinical samples. The on-disc PBMC segmentation and quantification process also made the study much user-friendly and automated while facilitating a short sample-toanswer time. Currently, we are investigating different microfluidic approaches to increase the PBMC extraction efficiency while making the cell segmentation and quantification procedure a single-step process by developing advanced cell-segmentation algorithm to contribute to further automation and efficiency of the PBMC quantification. Furthermore, through the addition of further sample processing steps, we target to achieve 3 parts or 5 parts differential WBC segmentation and quantification using the integrated microfluidic platform. There is also high potential for this biosensing platform to detect multiple biomarkers which can be achieved by splitting the extracted plasma on-disc into several samples followed by separately adding several specific antibody-functionalized MNBs into the metered samples leading to the quantification of multiple biomolecules in plasma along with total WBC count.

\section{Conflicts of Interest}

There are no conflicts of interests to declare.

\section{Acknowledgements}

This work was financially supported by the European Research Council under the European Union's Seventh Framework Program (FP7/2007-2013) grant no. 320535-HERMES. The authors also acknowledge the support from the IDUN project (grant no. DNRF122) funded by the Danish National Research Foundation and the Velux Foundations. The author thanks Dr. Robert Burger for his valuable advice on the disc design. 


\section{References}

[1] Pepys MB, and Hirschfield GM, 2003. C-reactive protein : a critical update. J Clin Invest 111, 1805-1812.

[2] Peltola H, and Jaakkola M, 1988. C-reactive protein in early detection of bacteremic versus viral infections in immunocompetent and compromised children. J Pediatr113, 641-646

[3] Coelho L, Almeida E, Fernandes A, Mealha R, Moreira P, and Sabino H, 2005. C-reactive protein as a marker of infection in critically ill patients Clin Microbiol 11, 101-108.

[4] Pfützner A FT., 2006. High-Sensitivity C-Reactive Protein as Cardiovascular Risk Marker in Patients with Diabetes Mellitus. Diabetes Technol Ther. 8, 28-37.

[5] Gussekloo J, Schaap MCL, Fro M, Blauw GJ, and Westendorp RGJ, 2000 Atherosclerosis and Lipoproteins C-Reactive Protein Is a Strong but Nonspecific Risk Factor of Fatal Stroke in Elderly Persons. Arter Thromb Vasc Biol. 20, 1047-1051.

[6] Lin X, Yu H, Zhao C, Qian Y, Hong D, Huang K, Mo J, Qin A, Fang X, and Fan S, 2016. The Peripheral Blood Mononuclear Cell Count Is Associated With Bone Health in Elderly Men. Medicine (Baltimore). 95, e3357.

[7] Todd JK, 1974. Childhood infections. Diagnostic Value of Peripheral White Blood Cell and Differential Cell Counts. Am J Dis Child. 127, 810 816.

[8] Xu W, Wu H, Ma S, Bai F, Hu W, Jin Y, and Liu H, 2013. Correlation between Peripheral White Blood Cell Counts and Hyperglycemic Emergencies. Int J Med Sci. 10, 758-765.

[9] Vozarova B, Weyer C, Lindsay RS, Pratley RE, Bogardus C, and Tataranni PA, 2002. High White Blood Cell Count Is Associated With a Worsening of Insulin Sensitivity and Predicts the Development of Type 2 Diabetes. Diabetes. 51, 455-461.

[10] Karthikeyan V, and Lip G, 2006. White blood cell count and hypertension. J Hum Hypertens. 20, $310-312$.

[11] Nakanishi N, Yoshida H, Matsuo Y, Suzuki K, and Tatara K, 2002. White blood-cell count and the risk of impaired fasting glucose or Type II diabetes in middle-aged Japanese men. Diabetologia 45, 42-48.

[12] Jee SH, Park JY, Kim H, Lee TY, and Samet JM, 2005. Original Contribution White Blood Cell Count and Risk for All-Cause, Cardiovascular and Cancer Mortality in a Cohort of Koreans. Am J Epidemiol. 162, 1062-1069.

[13] Farhangi MA, Keshavarz S, Eshraghian M, and Ostadrahimi A, 2013. White Blood Cell Count in Women : Relation to Inflammatory Biomarkers, Haematological Profiles, Visceral Adiposity, and Other Cardiovascular Risk Factors. J Heal Popul NUTR.31, 58-64.

[14] Brown DW, Giles WH, and Croft JB, 2001. White blood cell count : An independent predictor of coronary heart disease mortality among a national cohort. J Clin Epidemiol. 54, 316-322.

[15] Korppi M, and Leinonen M, 1997. White blood cells , C-reactive protein and erythrocyte sedi- mentation rate in pneumococcal pneumonia in children. Eur Respir J.10, 1125-1129.

[16] Haider DG, and Leuchten N, 2006. C-reactive protein is expressed and secreted by peripheral blood mononuclear cells. Clin Exp Immunol. 146 533-539.

[17] Allin KH, and Nordestgaard BG, 2011. Elevated C-reactive protein in the diagnosis, prognosis, and cause of cancer. Crit Rev Clin Lab Sci. 48, $155-170$.

[18] Lin G, Jiang X, Peng J, Xiao J, and Xia Z, 2014. Prognostic Significance of the Peripheral Blood Absolute Monocyte Count in Patients with Locally Advanced or Metastatic Hepatocellular Carcinoma Receiving Systemic Chemotherapy. Asian Pac J Cancer Prev. 15, 6387-6390.

[19] Price CP, Trull AK, Berry D, and Gorman EG, 1987. Development and validation of a particle-enhance turbidimetric immunoassay for Creactive protein. J. Immunol. Methods 99, 205-211.

[20] Rifai N, Tracy RP, and Ridker M,1999. Clinical efficacy of automated high-sensitivity C-reactive protein assay. Clin. Chem. 45, 2136-2141.

[21] Fock J, Parmvi M, Strömberg M, Svedlindh P, Donolato M, and Fougt M, 2017. Biosensors and Bioelectronics Comparison of optomagnetic and AC susceptibility readouts in a magnetic nanoparticle agglutination assay for detection of C-reactive protein detector. Biosens Bioelectron. $88,94-100$

[22] Zhu X, and Publicover NG, 2010. Magnetic bead based assay for C-reactive protein using quantum-dot fluorescence labeling and immunoaffinity separation. Analyst $135,381-389$.

[23] Tsai HY, Hsu CF, Chiu IW, and Bor FC, 2007. Detection of C-reactive protein based on immunoassay using antibody-conjugated magnetic nanoparticles. Anal Chem. 79, 8416-8419.

[24] Zhang P, Bao Y, Draz MS, Liu HLC, and Han H, 2015. Rapid and quantitative detection of C-reactive protein based on quantum dots and immunofiltration assay. Int J Nanomedicine.10, 6161-6173. 
[25] Ming C, Chen X, Xu P, Pen J, Zhu X, and Zhu D, 2014. Rapid and quantitative detection of C-reactive protein using quantum dots and immunochromatographic test strips. Int J Nanomedicine.9, 5619-5626.

[26] Centi S, Sanmartin LB, Tombelli S, Palchetti I, and Mascini M, 2009. Detection of C reactive protein (CRP) in serum by an electrochemical aptamer-based sandwich assay. Electroanalysis.21, 1309-1315

[27] Meyer MHF, Hartmann M, and Keusgen M, 2006. SPR-based immunosensor for the CRP detection - A new method to detect a well known protein. Biosens Bioelectron. 21, 1987-1990.

[28] Wu B, Jiang R, Wang Q, Huang J, Yang X, Wang K, Li W, Chen N, and Li Q, 2016. Detection of C-reactive protein using nanoparticleenhanced surface plasmon resonance using an aptamer-antibody sandwich assay. Chem Commun. 52, 3568-3571.

[29] Albrecht C, Kaeppel N, and Gauglitz G, 2008. Two immunoassay formats for fully automated CRP detection in human serum. Anal Bioanal Chem. 391:1845-1852.

[30] Chan LL, Laverty DJ, Smith T, Nejad P, Hei H, Gandhi R, Kuksin D, and Qiu J, 2013.. Accurate measurement of peripheral blood mononuclear cell concentration using image cytometry to eliminate RBC-induced counting error. J Immunol Methods.388, 25-32.

[31] Ouyang Y, and Li J, Haverstick DM and Lander JP, Rotation-driven microfluidic disc for while blood cell enumeration using magnetic bead aggregation. 2016. Anal. Chem. 88, 11046-11054.

[32] Moen ST, Hatcher CL, and Singh AK, 2016. A centrifugal microfluidic platform that separates whole blood samples into multiple removable fractions due to several discrete but continously density gradient sections. PLoS One 11, e0153137.

[33] Burger R, Kirby D, Glynn M, Nwankire C, O’Sullivan M, Siegrist J, Kinahan D, Aguirre G, Kijanka G, Gorkin R, and Ducrée J, 2012. Centfifugal microfluidics for cell analysis. Curr. Opin. Chem. Biol.16, 409-414.

[34] Turner APF, 2013. Biosensors: sense and sensability. Chem. Soc. Rev. 42, 3184-3196.

[35] English D, and Andersen BR, 1974. Single-step separation of red blood cells, granulocytes and mononuclear leukocytes on discontinuous density gradients of Ficoll-Hypaque. J Immunol Methods.5, 249-252.

[36] Quan X, Uddin R, Heiskanen A, Parmvi M, Nilson K, Donolato M, Hansen MF, Rena G, and Boisen A, 2015. The copper binding properties of metformin - QCM-D, XPS and nanobead agglomeration. Chem Commun.51, 17313-17316.

[37] Horng HE, Yang SY, Hong CY, Liu CM, Tsai PS, Yang HC, and Wu CC, 2006. Biofunctionalized magnetic nanoparticles for high-sensitivity immunomagnetic detection of human C-reactive protein. Appl Phys Lett.88, 252506.

[38] Wu SJ, Duan N, Wang ZP, and Wang HX, 2011. Aptamer-functionalized magnetic nanoparticle-based bioassay for the detection of ochratoxin a using upconversion nanoparticles as labels. Analyst 136, 2306-2314.

[39] Donolato M, Antunes P, de la Torre TZG, Hwu E-T, Chen C-H, Burger R, Rizzi G, Bosco FG, Stromme M, Boisen A, and Hansen MF, 2015. Quantification of rolling circle amplified DNA using magnetic nanobeads and a Blu-ray optical pick-up unit. Biosens Bioelectron. 67, 649655 .

[40] Uddin R, Burger R, Donolato M, Fock J, Creagh M, Hansen MF, and Boisen A, 2016. Lab-on-a-disc agglutination assay for protein detection by optomagnetic readout and optical imaging using nano- and micro-sized magnetic beads. Biosens Bioelectron.85, 351-357.

[41] Antunes P, Watterson D, Parmvi M, Burger R, Boisen A, Young P, Cooper MA, Hansen MF, Ranzoni A, and Donolato M, 2015. Quantification of NS1 dengue biomarker in serum via optomagnetic nanocluster detection. Sci Rep.5, 16145

[42] Hecht A, Commiskey P, Shah N, and Kopelman R, 2013. Bead assembly magnetorotation as a signal transduction method for protein detection Biosens Bioelectron. 48, 26-32.

[43] Yang J, Donolato M, Pinto A, Bosco FG, Hwu E-T, Chen C-H, Alstrom TS, Lee G-H, Schäfer T, Vavassori P, Boisen A, Lin Q, and Hansen MF, 2016. Blu-ray based optomagnetic aptasensor for detection of small molecules. Biosens Bioelectron.75, 396-403.

[44] Uddin R, Habiba NE, Rena G, Hwu E-T, and Boisen A, 2017. New evidence for the mechanism of action of a type-2 diabetes durg using a magnetic bead-based automated biosensing platform. ACS Sensors, DOI:10.1021/acssensors.7b00384.

[45] Fredborg M, Andersen KR, Jorgensen E, Droce a, Olesen T, Jensen BB, Rosenvinge FS, and Sondergaad TE, 2013. Real-time optical antimicrobial susceptibility testing. J Clin Microbiol.51, 2047-2053.

[46] Zhao Y, Schwemmer F, Zehnle S, von Stetten F, Zengerle R, and Paust N, 2015. Centrifugo-pneumatic sedimentation, re-suspension and transport of microparticles. Lab Chip. 1, 4133-4137.

[47] Mark D, Metz T, Haeberle S, Lutz S, Dcurée J, Zengerle R, and von Stetten F, 2009. Centrifugo-pneumatic valve for metering of highly wetting liquids on centrifugal microfluidic platforms. Lab Chip. 9, 3599-3603.

[48] Piper KE, Fernandez-Sampedro M, Steckelberg KE, Mandrekar JN, Karau MJ, Steckelberg JM, Berbari EF, Osmon DR, Hansen AD, Lewallen DG, Cofield RH, Sperling JW, Sanchez-Sotelo J, Huddleston PM, Dekutoski MB, Yaszemski M, Currier B, and Pater R, 2010. C-reactive protein, erythrocyte sedimentation rate and orthopedic implant infection. PLoS One. 5, e9358. 
[49] O'Reilly EJ, Conroy PJ, Hearty S, Keyes TE, O'Kennedy R, Forster RJ and Dennany L, 2015. Electrochemiluminiscence platform for the detection of Creactive proteins: application of recombinant antibody technology to cardiac biomarker detection. RSC Adv. 5, 67874-67877.

[50] Kinahan DJ, Kearney SM, Kilcawley NA, and Early PL, 2016. Density-Gradient Mediated Band Extraction of Leukocytes from Whole Blood Using Centrifugo-Pneumatic Siphon Valving on Centrifugal Microfluidic Discs. PLoS One 5, e0155545.

[51] Grievink HW, Luisman T, Kluft C, Moerland M, and Malone KE, 2016. Comparison of Three Isolation Techniques for Human. Biopreserv Biobank. 14, 410-415. 


\section{Biography}

Dr Rokon Uddin obtained his PhD degree in 2018 from the Department of Micro-and Nanotechnology, Technical University of Denmark under the supervision of Prof. Anja Boisen. His research works are focused on designing and development of microfluidic chips, relevant biomedical/biosensing instrumentation and bioassays for biomedical applications. His doctoral research work was based on the development of magnetic bead-based biochemical assays and integration of the assays on different microfluidics chips/discs for point-of-care application as well as for studying biochemical interactions (e.g. drug-metal interactions) through optical characterization. 
(a)

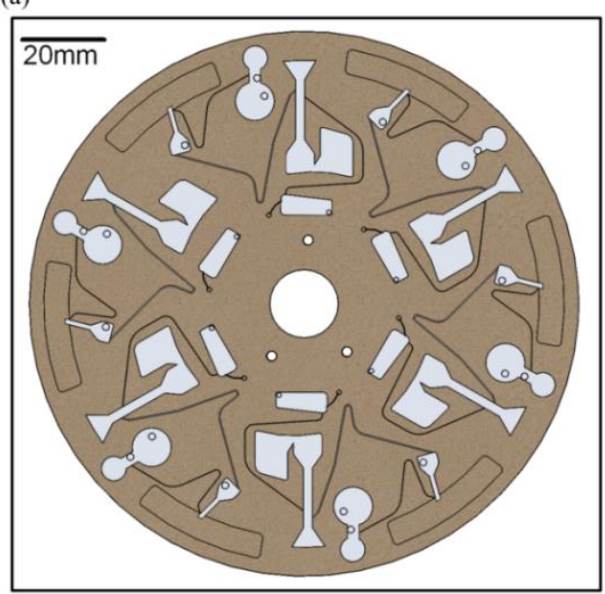

(b)

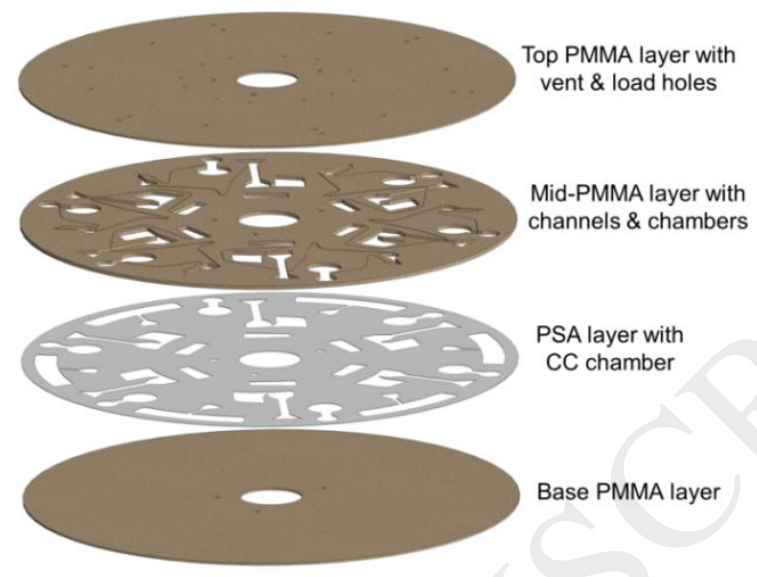

Fig. 1: (a) Schematic of the microfluidic disc with six repeating functional units. (b) Schematic of the four different layers of the disc. The top two PMMA layers were thermally bonded and subsequently bonded to the bottom layer using a structured PSA layer.
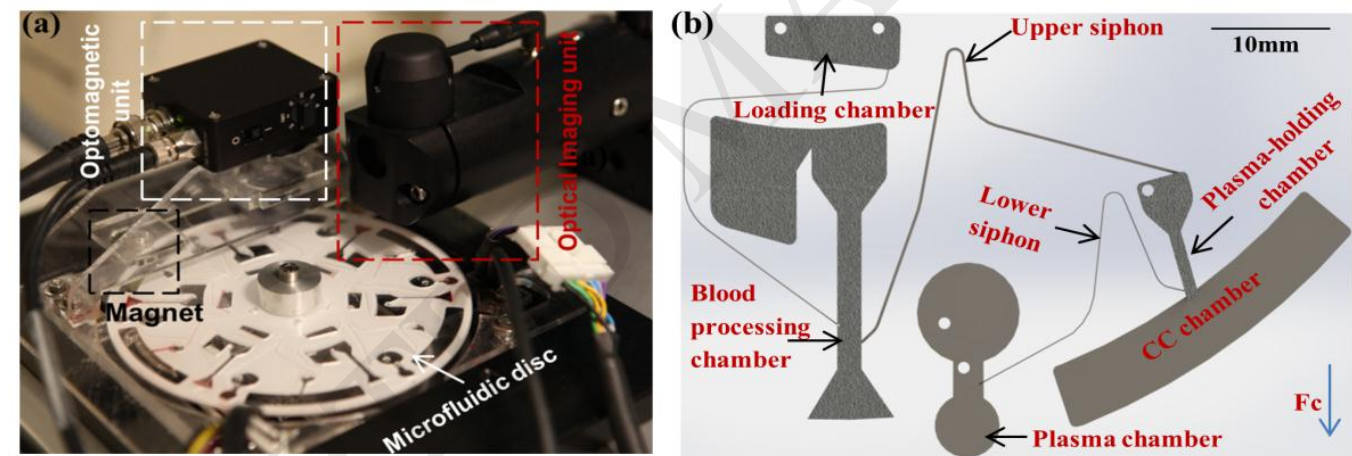

(c)
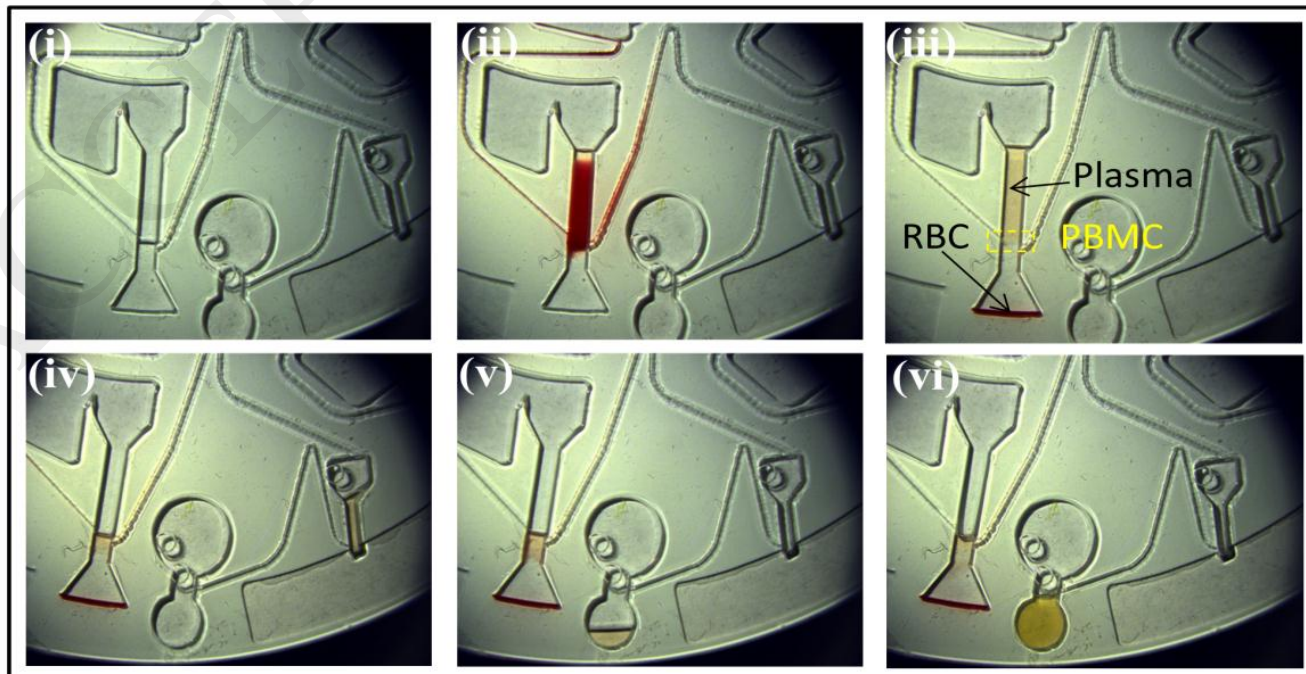
Fig. 2: (a) Experimental platform with the two integrated detector units. (b) A unit of the microfluidic disc demonstrating different chambers and channels; $F_{c}$ refers to centrifugal force (c) Sequential experimental images from sample loading to stratification and addition of MNBs to the extracted plasma: (i) Loading of DGM (ii) Overlaying of blood on DGM (iii) stratification into RBCs,

PBMCs and plasma due to centrifugation, (iv) Extraction of blood plasma and PBMC layer from the blood processing chamber after priming of upper siphon, (v) Transfer of plasma to the plasma chamber after lower siphon priming, (vi) Addition of MNBs to the extracted plasma for further processing.

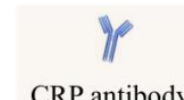

CRP antibody

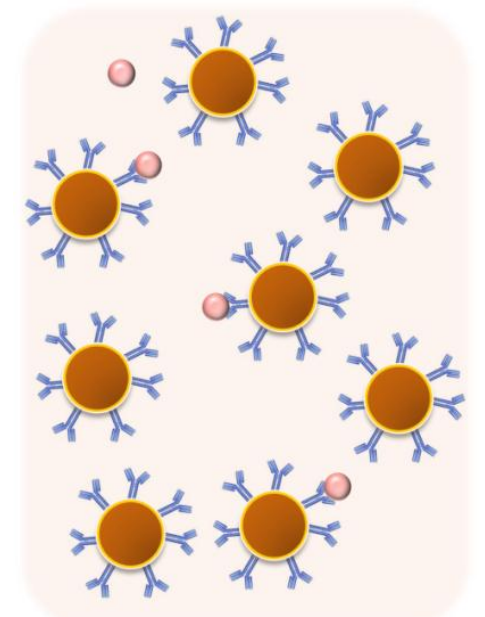

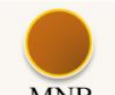

MNB

Fig. 3: The left panel of the image demonstrates the mixing and random distribution of antibody-functionalized MNBs and CRP antigen. The right panel illustrates that magnetic incubation facilitates the formation of CRP-bridged MNB clusters. 


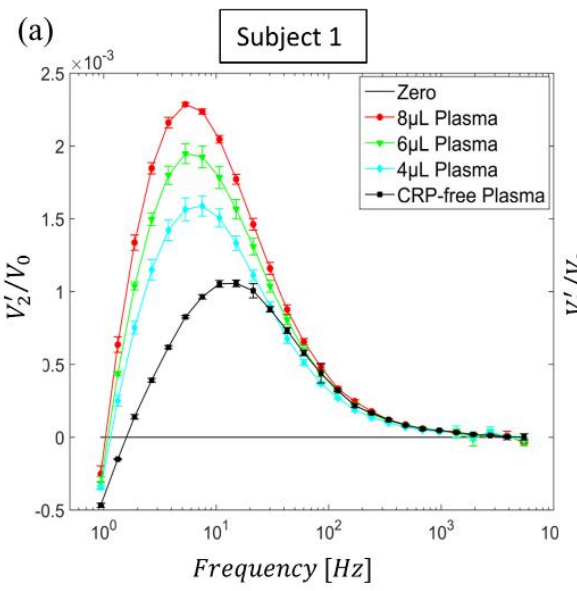

(d) (b)

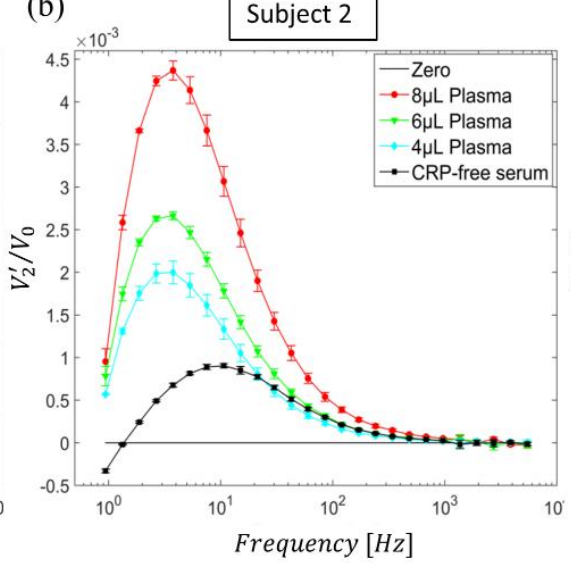

(c)

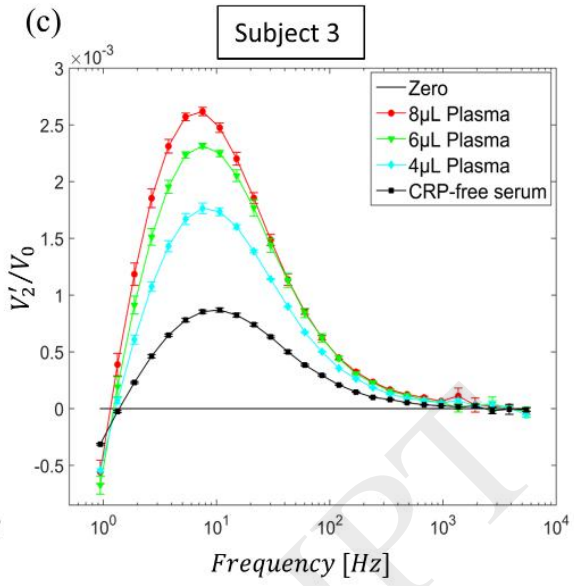

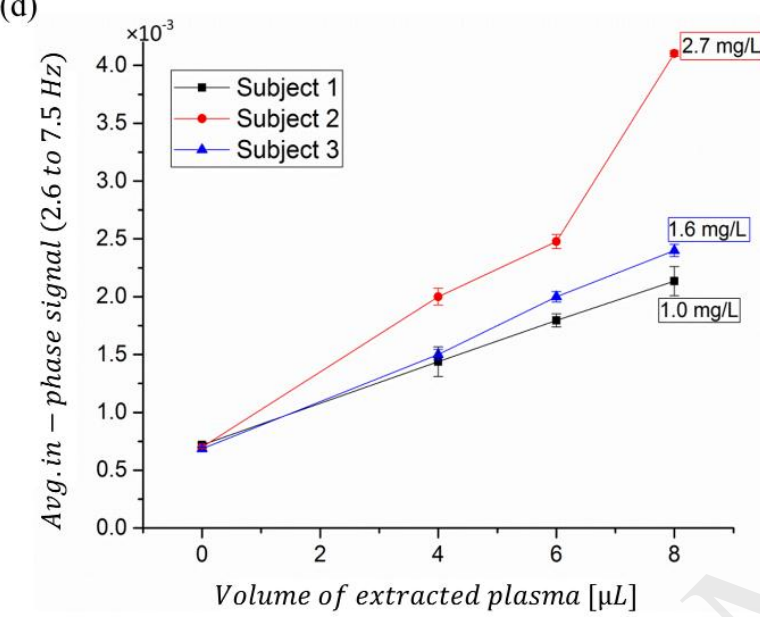

(e)

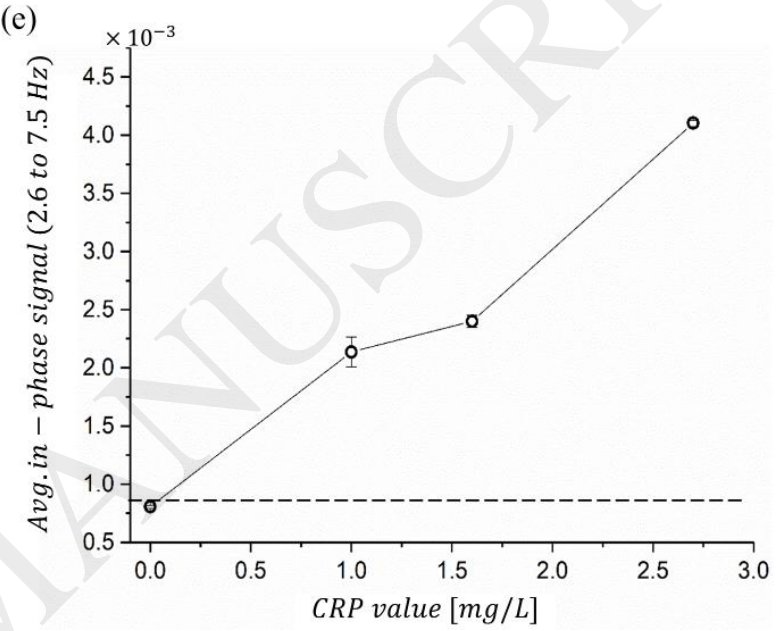

Fig. 4. (a), (b) and (c) Normalized in-phase optomagnetic signal, $V_{2}^{\prime} / V_{0}$, vs. frequency of the applied magnetic field indicating CRP concentrations of Subject 1, Subject 2 and Subject 3, respectively, for different amounts of extracted plasma. $8 \mu \mathrm{L}$ of extracted plasma refers to the CRP concentration of $1.0 \mathrm{mg} / \mathrm{L}, 2.7 \mathrm{mg} / \mathrm{L}$ and $1.6 \mathrm{mg} / \mathrm{L}$ for Subjects 1-3, respectively. (d) Average values of $V_{2}^{\prime} / V_{0}$ at $f=2.6 \mathrm{~Hz}$ to $7.5 \mathrm{~Hz}$ vs. relative amount of extracted plasma demonstrating the difference between the CRP level of the subjects. Error bars represent the standard deviation on the mean (SDOM) obtained from triplicate measurements. (e) Corresponding average values of optomagnetic signal (2.6-7.5 Hz) vs. measured CRP concentration for undiluted $8 \mu \mathrm{L}$ plasma samples. The black dotted line represents the mean blank signal plus the 3 times its standard deviation, which is used to estimate the concentration resolution. 
(a)

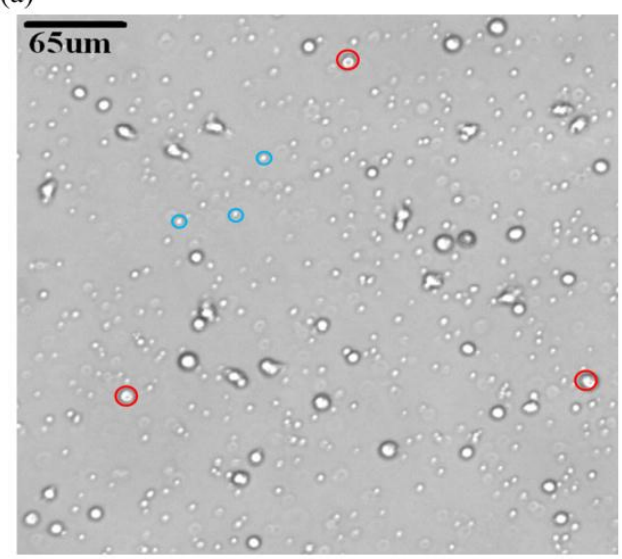

(c)

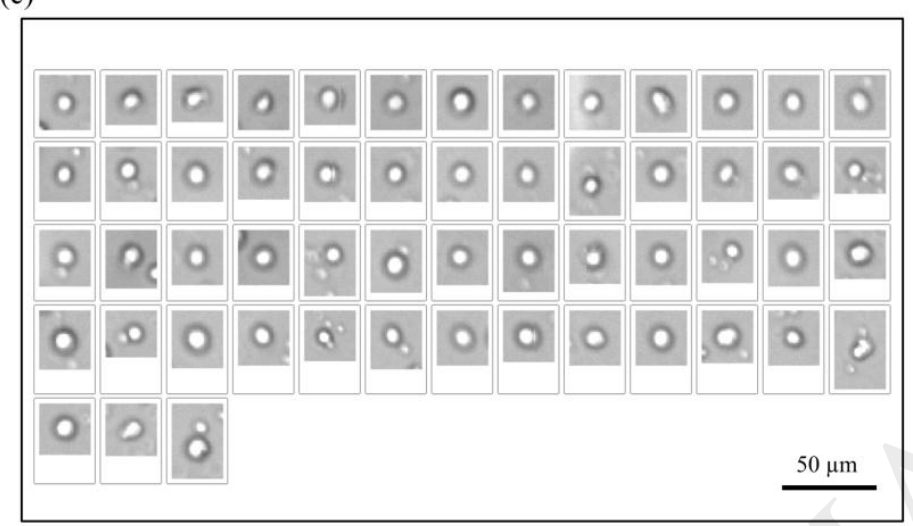

(b)

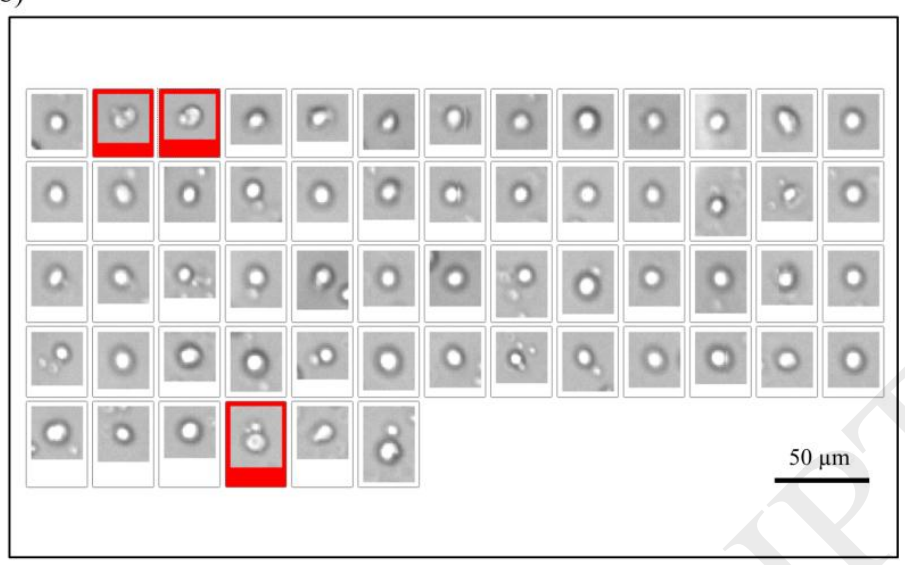

(d)

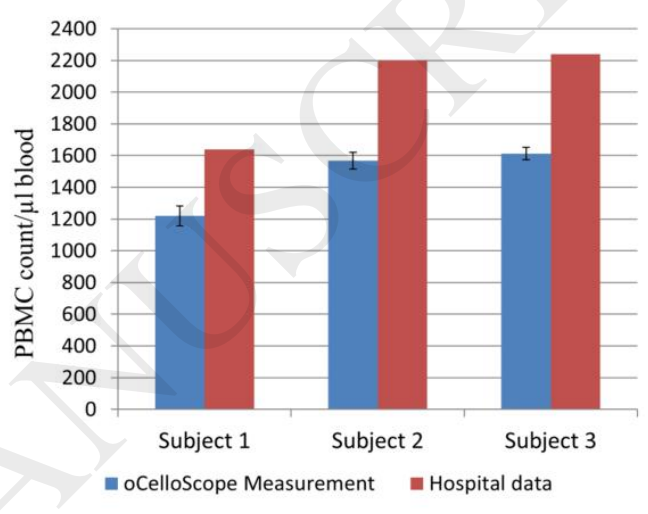

Fig. 5 (a) An experimental image of a part of the CC chamber on the microfluidic disc scanned by the oCelloScope. The red circles indicate the contamination of RBCs in the pool of PBMCs and platelets (blue-circled). (b) RBC contamination (red squares) after $1^{\text {st }}$ image segmentation step. (c) A view of the segmented PBMCs after final image segmentation which are quantified by Uniexplorer. (d) PBMC quantification from three different subjects demonstrating measurements obtained by oCelloScope and compared with the hospital data (obtained by SYSMEX measurement). Error bars represent the SDOM obtained from triplicate measurements. 